\title{
Hospitalização integral para tratamento dos transtornos alimentares: a experiência de um serviço especializado
}

\author{
Inpatient treatment for eating disorders: the \\ experience of a specialized service \\ Raphaela Fernanda Muniz Palma', José Ernesto dos Santos², Rosane Pilot Pessa Ribeiro 3
}

\section{RESUMO}

Objetivo: Descrever as características da hospitalização integral para o tratamento de transtornos alimentares em um serviço especializado de Ribeirão Preto, SP. Métodos: Foram revisados todos os prontuários dos pacientes em seguimento pelo serviço, de 1982 até 2011, especialmente aqueles que tiveram indicação de internação integral. Foram coletados dados sociodemográficos e referentes ao diagnóstico. Resultados: No período referido, 186 pacientes receberam atendimento pelo serviço e, desses, 44,6\% $(n=83)$ foram internados para tratamento. Ao longo do tempo, houve redução na relação atendimento/internação, passando de $77,7 \%$ para $36,2 \%$ dos casos. A média de internações foi de 1,9 $\pm 3,9$ vezes, e $73,5 \%$ ( $n$ = 61) dos pacientes foram hospitalizados apenas uma vez. A duração média da internação, independentemente do número de hospitalizações, foi de 70,6 115,9 dias (variação de 3 a 804 dias). A predominância foi do sexo feminino, raça branca, solteira, sem filhos e com idade média de 23,3 \pm 10,8 anos. O diagnóstico predominante foi de anorexia nervosa (85,5\%), sobretudo em seu tipo restritivo (54,2\%). As indicações mais frequentes para internação foram para realização de terapia nutricional (50,9\%), seguida da investigação do quadro clínico $(30,1 \%)$ e por causa de depressão e/ou ideação suicida (10,9\%). Conclusão: A hospitalização integral é uma modalidade terapêutica necessária para o tratamento desses quadros, e sua frequência foi considerada significativa, porém diminuiu ao longo do tempo. Esse resultado pode ser explicado pela tendência de desospitalização a partir da reforma psiquiátrica, do diagnóstico e tratamento mais precoces e da experiência adquirida pelos profissionais do serviço ao longo dos anos.

\section{ABSTRACT}

Objective: To describe the characteristics of inpatient treatment for eating disorders in a specialized service in Ribeirao Preto, SP. Methods: We reviewed all the medical records of patients followed up for the service between 1982 and 2011, particularly those who received inpatient treatment. Sociodemographic data and related to diagnosis were collected. Results: In the said period, 186 patients were treated by the service and $44.6 \%$ ( $n=83$ )

1 Universidade de São Paulo (USP), Escola de Enfermagem de Ribeirão Preto (EERP), Programa de Pós-Graduação em Enfermagem em Saúde Pública. 


\section{Keywords}

Eating disorders, inpatient

treatment, anorexia

nervosa, bulimia nervosa. were hospitalized for treatment. A reduction was observed in hospitalization rates of $77,7 \%$ to $36,2 \%$ over time. The average hospitalization was $1.9 \pm 3.9$ times of which $73.5 \%$ ( $n=$ 61) patients were hospitalized only once. The mean duration of hospitalization, regardless of the number of hospitalizations was $70.6 \pm 115.9$ days (range 3-804 days). Most patients were female, Caucasian, unmarried, childless and with a mean age of $23.3 \pm 10.8$ years old. The predominant diagnosis was anorexia nervosa (85.5\%), especially in its restrictive type (54.2\%). The most common indications for hospitalization were for nutritional support therapy (50.9\%), followed by the clinical research (30.1\%) and due to depression or suicidal ideation (10.9\%). Conclusion: Inpatient treatment was a therapeutic modality required for treatment of these patients and their frequency was significant, but decreased over time. This result can be explained by the trend of deinstitutionalization from the psychiatric reform, earlier diagnosis and treatment and the experience gained by service professionals over the years.

\section{INTRODUÇÃO}

Os transtornos alimentares (TA) são doenças graves e complexas cuja etiologia é multifatorial, envolvendo fatores genéticos, fisiológicos, psicológicos e ambientais. Dessa forma, torna-se imprescindível o olhar atento e multifacetado para o manejo terapêutico que se apresenta, quase sempre, bastante desafiador, requerendo abordagem de uma equipe multidisciplinar bem treinada em serviços especializados' Os TA mais conhecidos e discutidos são a anorexia nervosa (AN) e a bulimia nervosa (BN).

O nível de cuidado apropriado para o paciente é determinado no momento do seu diagnóstico inicial e, posteriormente, sempre que uma mudança relevante em sua condição exija transição para um nível diferente. As configurações incluem o seguimento ambulatorial, tratamento domiciliar, hospitalização parcial em hospitais-dia e hospitalização integral para pacientes graves que apresentam complicações agudas da doença. Ao determinar o nível inicial de atendimento ou a mudança para um nível diferente de cuidados, é essencial analisar em conjunto a condição física geral, complicações médicas, deficiências, comorbidade psiquiátrica, comportamento, interação familiar, recursos sociais, meio ambiente e serviços disponíveis ${ }^{2-4}$.

A hospitalização integral é a modalidade terapêutica utilizada pelas equipes quando outras tentativas de tratamento falharam ou não conseguem obter resultados satisfatórios. Ocorre em serviços especializados sob regime de internação em hospitais de nível terciário ou hospitais universitários. As indicações para hospitalização integral de pacientes com TA, em geral, englobam o estado nutricional insatisfatório, na maioria das vezes com peso abaixo de 75\% de adequação segundo o índice de massa corporal ou perda de 15\% do peso corporal em três meses; episódios de descompensação clínica com distúrbios hidroeletrolíticos; níveis de pressão arterial sistólica/diastólica baixos, inferior a 90/60 mmHg em adultos e 80/50 mmHg em crianças e adolescentes, frequência cardíaca menor que 50 bpm; pre- sença de comorbidades psiquiátricas ou risco de suicídio iminente; pouca continência familiar e situações de tensão ambiental; e também abuso de substâncias e exacerbação de sintomas purgativos ${ }^{5,6}$.

No início dos anos 1980, foi criado pela Divisão de Nutrologia do Departamento de Clínica Médica da Faculdade de Medicina de Ribeirão o Grupo de Assistência em Transtornos Alimentares (GRATA) para prestar assistência a portadores de TA no Hospital das Clínicas (HCFMRP-USP). Ao longo dos anos, à sua equipe agregaram-se vários profissionais e, atualmente, o serviço conta com uma equipe multidisciplinar composta por médicos (nutrólogo e psiquiatra), psicólogos e nutricionistas, além de estagiários nas áreas de psicologia e nutrição ${ }^{7}$.

O serviço oferece atendimento semanal na esfera ambulatorial, com frequência de retorno variada, dependendo da necessidade do caso. São oferecidos atendimentos clínicos individuais com médicos nutrólogos e psiquiatras, consultas com nutricionistas, apoio psicológico, além da psicoterapia individual para pacientes e familiares. Atividades educativas/ informativas e de apoio em grupo de natureza psicológica e nutricional também são realizadas antes dos atendimentos para pacientes e familiares separadamente. Os pacientes que necessitam de intervenção hospitalar para tratamento e/ou estabilização do quadro clínico são encaminhados para internação nas enfermarias de Clínica Médica (Unidade Metabólica) ou Psiquiatria no HCFMRP-USP. A admissão hospitalar é mediada pelos médicos que compõem a equipe do GRATA, geralmente residentes ou médicos contratados do HCFMRP-USP; o seguimento dos pacientes nessa situação é realizado pela equipe fixa de profissionais do hospital, com possibilidade de assistência de outras especialidades como terapeutas ocupacionais e assistentes sociais por pedido de interconsulta ${ }^{8}$.

O objetivo do presente estudo foi descrever as indicações e as características da hospitalização integral para tratamento de pacientes com transtornos alimentares seguidos por um serviço especializado desde o início do seu funcionamento. 


\section{MÉTODOS}

Trata-se de um estudo transversal de caráter descritivo e exploratório com delineamento quantitativo.

Inicialmente, foi realizado levantamento no sistema informatizado do hospital junto ao Departamento de Seção de Dados Médicos dos registros de pacientes que foram internados entre 1982 e 2011 com diagnóstico de AN ou BN.

Foram incluídos no estudo pacientes com diagnóstico de AN ou BN seguidos pelo GRATA, que foram internados no período referido, independentemente do sexo e idade, com duração de internação igual ou superior a três dias. Foram excluídos do estudo aqueles pacientes que, apesar do diagnóstico do TA, não receberam atendimento pelo GRATA ou cujos prontuários não foram encontrados nos arquivos médicos.

Os dados foram coletados a partir da revisão dos prontuários dos pacientes, seguindo o instrumento de coleta desenvolvido para a pesquisa. Foram colhidos dados sociodemográficos, da doença (tempo de sintomas e idade no diagnóstico e internação, tempo de tratamento da doença antes da internação) e informações relacionadas às internações (número, duração e indicação das internações). A definição das faixas etárias de crianças e adolescentes seguiu a classificação da Organização Mundial da Saúde9: crianças até 10 anos de idade e adolescentes entre 10 e 19 anos. Os pacientes adultos compreenderam aqueles com idade superior a 19 anos.

As informações foram transportadas para um banco de dados com técnica de dupla-digitação e validação no programa Excel do software Microsoft Office, versão 2007. Os resultados descritivos foram obtidos por meio do cálculo das médias (desvio-padrão), valores mínimos e máximos para as variáveis contínuas, além das frequências para as variáveis categóricas. Foi empregado o teste para análise de variância (ANOVA) e utilizou-se o método Bonferroni para verificar diferenças estatísticas entre as múltiplas comparações nesse teste. O valor de $\mathrm{p}<0,05$ foi adotado como significante e todos os cálculos das análises estatísticas foram realizados com auxílio do software SPSS versão 17.0 (SPSS Inc. Chicago, USA).

A pesquisa foi aprovada pelo Comitê de Ética em Pesquisa do HCFMRP-USP (no 976/2010).

\section{RESULTADOS}

No período de abrangência do estudo, o GRATA prestou atendimento para 186 pacientes com TA; 83 (44,6\%) deles necessitaram de, no mínimo, uma internação durante o tratamento.

Ao longo dos anos, constatou-se que a escolha da hospitalização como modalidade terapêutica foi diminuindo (Figura 1). No início do funcionamento do serviço, na década de $1980,77,7 \%$ dos pacientes ( $n=14$ ) em tratamento foram internados. Já na década seguinte, a relação entre pacientes atendidos e internados caiu para a metade $(n=28)$, e, a partir de 2000, a internação ocorreu em apenas 36,2\% dos casos (n $=41$ ) em seguimento.

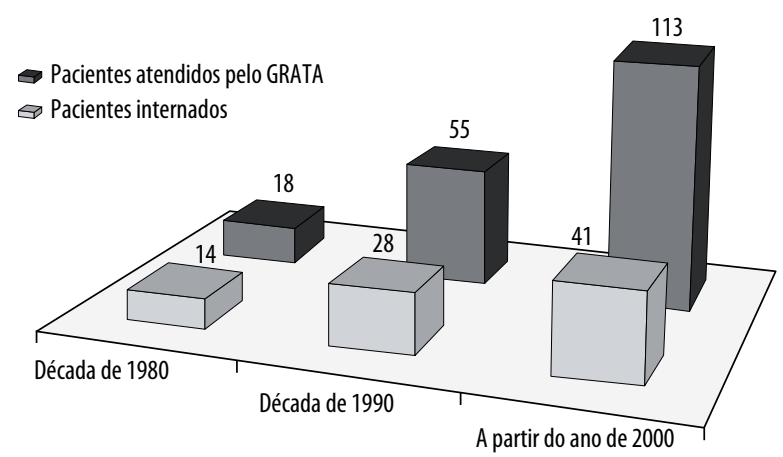

Figura 1. Número de pacientes atendidos pelo GRATA (Grupo de Assistência em Transtornos Alimentares, $n=186$ ) no período do estudo que necessitaram de internação $(n=83)$ durante o tratamento, segundo década. Ribeirão Preto, 2011.

Com relação ao número de internações, a média foi de $1,9 \pm 3,9$ vezes (com variação de 1 a 26 vezes). Constatou-se que a maioria dos pacientes que necessitaram de intervenção hospitalar para tratamento $(n=61 ; 73,5 \%)$ foi internada apenas uma vez; 18 pacientes $(21,7 \%)$ tiveram de duas a cinco internações; e apenas quatro pacientes $(4,8 \%)$ necessitaram de mais de cinco internações.

A média de idade na admissão hospitalar foi de 23,3 \pm 10,8 anos (variação de 8 a 58 anos), aumentando naturalmente com o decorrer do tempo para 30,9 $\pm 16,6$ anos (variação de 9 a 68 anos) na última internação daqueles que foram internados mais de uma vez.

Na primeira $(n=83)$ havia duas crianças $(2,4 \%), 37$ adolescentes (44,6\%) e 44 adultos (53\%); para aqueles que tiveram mais de uma internação $(n=22)$, a proporção na última internação foi de duas crianças (9\%), nove adolescentes (41\%) e 11 adultos (50\%).

O tempo médio total de hospitalização, independentemente do número de internações, foi de 70,6 \pm 115,9 dias, com extensa variação (3 a 804 dias). A duração da internação daqueles hospitalizados apenas uma vez $(n=61)$ foi de $41 \pm$ 37,6 dias (mínimo 3; máximo 159 dias) e para aqueles hospitalizados mais vezes $(n=22)$, somando-se todas as internações, a duração média foi de $152 \pm 199$ dias (mínimo 15, máximo 804 dias).

As indicações para internação mais frequentes foram para realização de terapia nutricional (suporte nutricional ou reposição hidroeletrolítica), seguida da investigação do quadro clínico e por causa de depressão e/ou ideação suicida (Figura 2). 


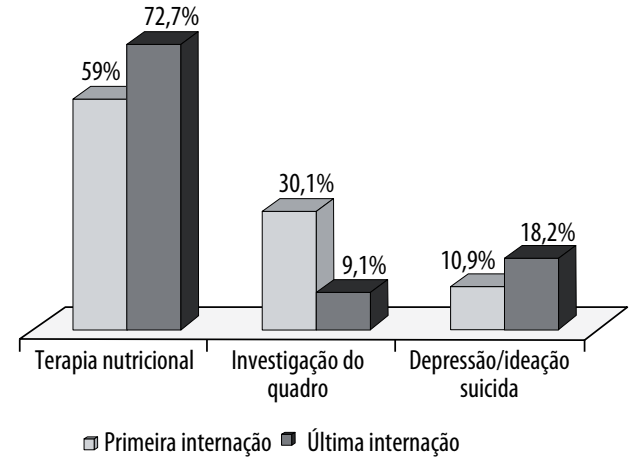

Figura 2. Número de pacientes hospitalizados com transtornos alimentares no HCFMRP-USP (Hospital das Clínicas da Faculdade de Medicina de Ribeirão Preto - Universidade de São Paulo), segundo indicação na primeira $(n=83)$ e última internação ( $n=22)$, Ribeirão Preto, 2011.

Aproximadamente um terço dos pacientes $(30,1 \%)$ em sua primeira hospitalização foi internado para investigação do quadro, número que naturalmente caiu na última internação. A necessidade de internação de pacientes por causa de depressão/ideação suicida ocorreu em poucos casos, e em análise mais detalhada observou-se que foi proporcionalmente maior entre pacientes com BN internados apenas uma vez.

A tabela 1 apresenta a distribuição da amostra segundo as características sociodemográficas. Evidencia-se predominância de pacientes do sexo feminino e da raça branca, com média de idade na época do diagnóstico de 22,6 \pm 10,7 anos, variando de 8 a 58 anos, sendo $53 \%$ adultos $(n=44)$ e $47 \%$ crianças e adolescentes $(n=39)$. A maioria era solteira, sem filhos, e cerca da metade dos pacientes era estudante cursando o ensino médio. Observa-se que a maior parte deles reside em cidades do estado de São Paulo, com cerca de um terço na cidade de Ribeirão Preto, SP.

A distribuição dos pacientes segundo diagnóstico do TA (Tabela 2) demonstrou preponderância de AN ( $n=71$; $85,5 \%$ ). Quando observados os seus subtipos, aproximadamente metade deles apresentava anorexia nervosa do tipo restritivo (AN-R), em menor escala apresentaram anorexia nervosa do tipo compulsão periódica/purgativo (AN-CP). Pacientes portadores de BN abarcaram a minoria dos casos. Em relação à idade na época do diagnóstico, essa foi semelhante entre os portadores de AN-R e AN-CP e maior naqueles com $\mathrm{BN}$, sem diferença estatística.

O tempo dos sintomas da doença antes do diagnóstico foi de 32,4 \pm 45,8 meses, com extensa variação de 1 a 240 meses. Quando separados diagnósticos e subtipos, observou-se que o tempo de sintomas dos pacientes com AN-R foi menor, e para os portadores de AN-CP ou BN o período sintomatológico foi, pelo menos, três vezes maior. O tempo de sintomas apresentados pelos pacientes com AN-R diferiu significativamente daqueles com AN-CP e BN $(p<0,05)$.
Tabela 1. Caracterização sociodemográfica dos pacientes hospitalizados com transtornos alimentares $(n=83)$, Ribeirão Preto, 2011

\begin{tabular}{lc}
\hline Variáveis & [n(\%)] \\
\hline Sexo & \\
Feminino & $79(95,2)$ \\
Masculino & $4(4,8)$ \\
Estado civil & \\
Solteiro & $63(76)$ \\
Casado/amasiado & $16(19,3)$ \\
Divorciado/viúvo & $4(4,7)$ \\
Filhos & \\
Sim & $18(21,7)$ \\
Não & $65(78,3)$ \\
Raça & \\
Branca & $78(94)$ \\
Mulata & $4(4,8)$ \\
Negra & $1(1,2)$ \\
Escolaridade & \\
Ensino fundamental & $21(25,3)$ \\
Ensino médio & $42(50,6)$ \\
Ensino superior & $20(24,1)$ \\
Procedência & \\
Ribeirão Preto & $30(36,1)$ \\
Outras cidades do Estado de São Paulo & $43(51,8)$ \\
Outros Estados & $10(12,1)$ \\
Ocupação & \\
Estudante & $46(55,4)$ \\
Do lar & $18(21,7)$ \\
Exerce atividades profissionais & $19(22,9)$ \\
\hline
\end{tabular}

Tabela 2. Caracterização dos pacientes hospitalizados com TA $(n=83)$, segundo o diagnóstico e subtipos, Ribeirão Preto, 2011

\begin{tabular}{|c|c|c|c|c|c|}
\hline & $\mathrm{n}(\%)$ & $\bar{x}$ & $\pm \mathrm{DP}$ & Mín & Máx \\
\hline AN-R & $45(54,2)$ & & & & \\
\hline Idade (anos) & & 21,7 & 12,6 & 8 & 58 \\
\hline Tempo de sintomas (meses) & & $15,4^{*}$ & 18,7 & 1 & 108 \\
\hline $\begin{array}{l}\text { Tempo de tratamento antes } \\
\text { da internação (meses) }\end{array}$ & & 3,4 & 17,1 & 1 & 30 \\
\hline $\mathrm{AN}-\mathrm{CP}$ & $26(31,3)$ & & & & \\
\hline Idade (anos) & & 23,4 & 9,4 & 12 & 52 \\
\hline Tempo de sintomas (meses) & & $45,1^{*}$ & 56,8 & 2 & 240 \\
\hline $\begin{array}{l}\text { Tempo de tratamento antes } \\
\text { da internação (meses) }\end{array}$ & & 8,3 & 19,9 & 1 & 96 \\
\hline BN & $12(14,5)$ & & & & \\
\hline Idade (anos) & & 24 & 3,9 & 19 & 32 \\
\hline Tempo de sintomas (meses) & & $68,2^{*}$ & 62,9 & 1 & 180 \\
\hline $\begin{array}{l}\text { Tempo de tratamento antes } \\
\text { da internação (meses) }\end{array}$ & & 1,6 & 3,6 & 1 & 12 \\
\hline
\end{tabular}

O tempo de tratamento para o TA entre o diagnóstico e a primeira internação teve média de 4,8 $\pm 12,5$ meses e variação de 1 a 96 meses. O maior tempo de tratamento antes da internação ocorreu entre pacientes com AN-CP, evidenciado tanto na média como no tempo máximo apresentado, seguidos de pacientes com AN-R. Os portadores de BN apre- 
sentaram os intervalos mais curtos de tratamento antes da internação. Não foram identificadas diferenças significativas entre as médias de tempo de tratamento antes da internação nos diferentes diagnósticos.

\section{DISCUSSÃO}

Os quadros mais graves de TA podem levar à necessidade de internação para tratamento durante o curso da doença, na tentativa de reverter ou minimizar as complicações clínicas e comorbidades associadas. Entretanto, pouco se sabe sobre a relação de pacientes atendidos e internados nos serviços especializados. Os resultados na literatura referem-se às descrições específicas dos pacientes internados. Casper e Jabine ${ }^{10}$ publicaram que $88 \%$ dos pacientes necessitaram de pelo menos uma internação no período de oito anos após diagnóstico do TA. A frequência de internação entre os pacientes seguidos pelo GRATA nos seus 30 anos de serviço foi de 44,6\%, índice considerado representativo, pois quase metade dos pacientes foi internada no mínimo uma vez para tratamento da doença. Na literatura, há escassez de dados sobre as características dos pacientes com TA internados e o tipo de tratamento prestado ${ }^{11}$.

Wiseman et al. ${ }^{12}$, em estudo sobre a hospitalização de pacientes com TA em Nova lorque, observaram mudança nos padrões entre 1980 e 1998, com aumento no número de hospitalizações, passando de 20 para 182. A experiência de um centro de tratamento especializado em TA em Denver, também nos Estados Unidos, demonstrou que, apenas entre os anos de 2008 e 2010, 76 pacientes foram admitidos para tratamento hospitalar ${ }^{13}$. No GRATA, apesar de evidenciado aumento no número de hospitalizações com o passar dos anos, observou-se que a escolha de hospitalização como modalidade terapêutica diminuiu nos últimos 30 anos. Houve redução na relação atendimento/internação, passando de $77,7 \%$ para $36,2 \%$ dos casos.

Nas últimas décadas, o declínio na frequência de internações por TA tem sido observado em vários países. Na Polônia, entre 2003 e 2007, os hospitais psiquiátricos apresentaram redução de 5\% nesse índice, e nos hospitais gerais a queda foi de $14 \%^{14}$. Nos Estados Unidos, o mesmo cenário foi notado, visto que o tratamento hospitalar para TA ocorre apenas em casos crônicos, abrangendo principalmente a estabilização (suporte nutricional e hidratação) dos episódios agudos. Ao longo do tempo, com o aumento nos custos das internações, somado ao fato de que pacientes crônicos são frequentemente passíveis de readmissão e/ou necessitam de longos períodos de hospitalização, muitas companhias de planos de saúde passaram a não cobrir os custos da internação, tratando os pacientes em ambulatório ${ }^{12}$.

A redução dessa modalidade de tratamento para TA também pode ser vista como reflexo das novas políticas de saú- de, fruto do movimento da reforma psiquiátrica. Esse movimento tomou força na segunda metade da década de 1970, com objetivo de modificar o sistema de tratamento clínico da doença mental ${ }^{15}$.

No Brasil, o tratamento hospitalar para essas doenças sempre foi, em sua grande maioria, disponibilizado por grupos assistenciais em centros universitários vinculados a hospitais públicos com profissionais prestando serviços de forma voluntária ${ }^{16}$. A diminuição dessas internações pode estar associada ao fato de que, anteriormente, o tratamento hospitalar era imediatamente indicado, dado o acometimento físico e clínico dos pacientes que se encontravam quase sempre, muito debilitados. Com o passar dos anos, o melhor reconhecimento desses quadros pelos profissionais de saúde e sociedade como um todo favoreceu o diagnóstico e o tratamento precoces, evitando a exacerbação dos sintomas. Dessa forma, a abordagem terapêutica utilizando seguimento ambulatorial ou hospital-dia foi possível para a recuperação dos pacientes. Outro aspecto a ser considerado é a experiência adquirida pelos profissionais dos serviços para o manejo dos casos mais graves, o que também contribuiu para diminuição da hospitalização integral em primeira instância ${ }^{17-19}$. As indicações para hospitalização, desde o início do funcionamento do serviço até os dias atuais, seguem critérios clínicos claramente estabelecidos, por exemplo: peso corporal abaixo de 75\% do esperado, bradicardia, hipotensão, distúrbios hidroeletrolíticos graves, comprometimento orgânico, falta de suporte ambiental e/ou risco de suicídio ${ }^{2,20}$.

As características sociodemográficas dos pacientes hospitalizados pelo GRATA foram semelhantes em relação a outros estudos ${ }^{11-14,21}$.

A AN, por causa de suas graves consequências nos sistemas orgânicos decorrentes da desnutrição, é o diagnóstico de TA mais prevalente nas intervenções hospitalares ${ }^{22-24}$. A distribuição dos pacientes internados pelo GRATA de acordo com o diagnóstico também demonstrou preponderância de AN, sobretudo em seu subtipo restritivo.

Os estudos da literatura científica apontam que pacientes com AN são mais jovens. No presente estudo, a média de idade mais baixa foi encontrada entre pacientes com AN (21,7 anos). Entretanto, esse valor é superior às médias demonstradas em outros estudos, que variaram de 10 a 20,7 $\operatorname{anos}^{11,21,22,23,25,26}$.

O tempo de sintomas apresentado pelos pacientes até que eles sejam diagnosticados é bastante variável. As perturbações psicológicas que comumente se apresentam, próprias do quadro, passam comumente despercebidas em primeira instância pela família, por serem associadas às características típicas da adolescência, tais como: irritabilidade, alteração no caráter, humor depressivo, isolamento, ou em alguns casos, hiperatividade ${ }^{27}$. A instalação do quadro de TA pode ser inicialmente dissimulada com justificativas que não despertam intensa preocupação, visto que atualmente um 
regime alimentar com limitações quantitativas e qualitativas dos alimentos é encarado como atitude normal ${ }^{28}$. Diante de qualquer alegação referente à perda de peso, é comum os pacientes explicarem sua atitude pelo desejo de emagrecer, pois encaram-se demasiadamente gordos, mesmo apresentando-se aos olhos alheios uma magreza impressionante. A negação da doença, parte integrante do quadro dos TA, bem como a inadmissível recuperação do peso principalmente entre os anoréxicos, faz com que a procura pelo tratamento assuma uma tendência tardia ${ }^{28}$.

A despeito dos esforços para mascarar a doença, os TA acabam sendo reconhecidos com grande variação no tempo de sintomas até o diagnóstico: são detectados mais rapidamente em portadores de $\mathrm{AN}$, dado o progressivo e rápido emagrecimento, e tardiamente entre pacientes com BN, que comumente mantêm o peso dentro da faixa de normalidade. A pesquisa de Hudson et al. ${ }^{25}$ revelou que o tempo médio de sintomas apresentados por pacientes com AN foi de 1,7 ano e na BN, de 8,3 anos. Os achados do presente estudo, quando comparados aos desse autor, indicam tempo médio de sintomas menores, sendo 15,4 meses (1,3 ano) para pacientes com AN-R; 45,1 meses (3,7 anos) na AN-CP e 68,2 meses (5,7 anos) nos casos de BN.

Apesar de as hospitalizações ocorrerem mais frequentemente e em menor tempo nos pacientes com $\mathrm{AN}^{12,13,21,29}$, os achados do presente estudo demonstram que aqueles com $\mathrm{BN}$ apresentaram os intervalos mais curtos de tratamento antes da internação (1,6 $\pm 3,6$ meses). A inversão observada nessa amostra pode ser explicada pelo fato de que alguns desses pacientes foram internados para avaliação do quadro clínico e definição do diagnóstico. A maior parte deles foi internada em menor espaço de tempo, em virtude de distúrbios hidroeletrolíticos e complicações clínicas associadas aos mecanismos compensatórios recorrentes para evitar o ganho de peso, como indução de vômitos e abuso de laxantes.

A maioria dos pacientes deste estudo foi internada para receber terapia nutricional, objetivando a recuperação do peso e compensação dos distúrbios hidroeletrolíticos. As taxas de internação do presente estudo foram semelhantes àquelas descritas por Chu et al. ${ }^{13}$, em que $71 \%$ dos pacientes foram internados para estabilização médica no ACUTE.

Indicações para internação objetivando a investigação do quadro e diagnóstico estiveram presentes em aproximadamente um terço dos casos na admissão hospitalar, número que naturalmente caiu na última internação. Parte deles tinha diagnóstico de BN, o que pode explicar o fato de esses pacientes terem sidos submetidos a internações mais curtas (média 1,6 mês) do que os portadores de AN. As internações devidas à depressão/ideação suicida aumentaram na última internação entre aqueles mais graves que necessitaram de mais de uma internação. A depressão é uma comorbidade psiquiátrica que deve ser considerada e tratada por causa de sua associação direta com o suicídio. Estudos mostram que sua prevalência pode ser alta entre pacientes com TA ${ }^{11,22}$.
Pesquisas que adotam como metodologia a coleta de dados por meio de revisão de prontuários apresentam, inevitavelmente, viés importante relacionado à perda ou omissão de informações registradas pelos profissionais de saúde. Essa foi a principal dificuldade encontrada no desenvolvimento deste estudo, cuja perda amostral variou de 1,2\% a 9\% nas diferentes variáveis estudadas.

Dados sobre a hospitalização integral de pacientes portadores de AN ou BN são pouco conhecidos. No Brasil, isso se deve ao fato de existirem poucos serviços especializados que oferecem tratamento, sobretudo hospitalar, com predomínio de atividades de natureza assistencial. Consequentemente, investigações científicas nessa área apresentam limitações relacionadas às dificuldades na formação e treinamento de pesquisadores, além da falta de investimentos em recursos humanos, pois a maioria dos serviços conta com profissionais que atuam de forma voluntária. Esses fatores certamente contribuem para o pequeno conhecimento científico sobre o tema.

\section{CONCLUSÃO}

Conclui-se que a hospitalização integral é uma modalidade terapêutica necessária para o tratamento de anorexia ou bulimia nervosa, e a frequência de internação do serviço foi considerada significativa, porém diminuiu ao longo do tempo. A maioria dos pacientes foi internada apenas uma vez e era composta de mulheres brancas, adultas jovens, solteiras e portadoras de anorexia nervosa, sobretudo em seu tipo restritivo. A indicação mais frequente de internação dos pacientes foi para realização de terapia nutricional. Os resultados encontrados são compatíveis com outros dados da literatura. A diminuição da frequência de internação pode ser resultado da tendência de desospitalização para as doenças mentais como um todo a partir da reforma psiquiátrica, da divulgação pela mídia e do maior reconhecimento desses quadros pela sociedade em geral e pelos profissionais de saúde, favorecendo o diagnóstico e o tratamento mais precoces. Além disso, a experiência adquirida pelos profissionais do serviço no manejo dos casos mais graves possibilitou que o seguimento fosse dado em nível ambulatorial e hospital-dia. Porém, futuros estudos são necessários para comprovar essa hipótese.

\section{CONTRIBUIÇÕES INDIVIDUAIS}

Raphaela Fernanda Muniz Palma - Participou da concepção e desenho do estudo e foi responsável pela elaboração do artigo, além da coleta, análise e interpretação dos dados, aprovando sua versão final para publicação.

Rosane Pilot Pessa Ribeiro - Contribuiu significativamente na concepção e no desenho do estudo e orientou a análise, 
a interpretação dos dados e a elaboração do artigo, aprovando sua versão final para publicação.

José Ernesto dos Santos - Contribuiu significativamente na concepção e no desenho do estudo, revisou criticamente seu conteúdo intelectual e aprovou sua versão final para publicação.

\section{AGRADECIMENTOS}

À Profa. Dra. Cláudia Benedita dos Santos pela assessoria nas análises estatísticas e à Fundação de Amparo à Pesquisa do Estado de São Paulo (Fapesp) pelo auxílio financeiro para execução do projeto (processo no 2010/02417-8).

\section{CONFLITOS DE INTERESSE}

Os autores do manuscrito - Raphaela Fernanda Muniz Palma, Prof. Dr. José Ernesto dos Santos e Profa. Dra. Rosane Pilot Pessa Ribeiro - não possuem conflitos de interesse a serem declarados.

\section{REFERÊNCIAS}

1. Cartwright MM. Eating disorder emergencies: understanding the medical complexities of the hospitalized eating disordered patient. Crit Care Nurs Clin North Am. 2004;16:515-30.

2. Guimarães DBS, Salzano FT, Abreu CN. Indicações para internação hospitalar completa ou parcial. Rev Bras Psiquiatr. 2002;24(3):60-2.

3. Cabrera CC. Estratégias de intervenção interdisciplinar no cuidado com o paciente com transtorno alimentar: o tratamento farmacológico. Medicina. 2006;39(3):375-80.

4. Donini LM, Cuzzolaro M, Spera G, Badiali M, Basso N, Bollea MR, et al. Obesity and eating disorders. Indications for the different levels of care. An Italian Expert Consensus Document. Eat Weight Disord. 2010;15(Suppl 1-2):1-31.

5. Anzai N, Lindsey-Duddley K, Bidwell RJ. Inpatient and partial hospital treatment for adolescent eating disorder. Child Adolesc Psychiatr Clin N Am. 2002;11:279-309.

6. Woodside DB. Inpatient treatment and medical management of anorexia nervosa and bulimia nervosa. In: Fairburn CG, Brownell KD, editors. Eating disorders and obesity: a comprehensive handbook. 2nd ed. New York: The Guildford Press; 2002.

7. Dos Santos JE. GRATA: nossa história, trabalho e desafios. Medicina. 2006;39(3):323-6.

8. Da Silva LM, Dos Santos MA. Construindo pontes: relato de uma equipe multidisciplinar em transtornos alimentares. Medicina. 2006;39(3):415-24.

9. Organização Mundial da Saúde. Organização Mundial da Saúde (1965). Problemas de la salud de la adolescencia. Informe de un comité de expertos de la OMS. Informe técnico n³08. 1965; 30p.
10. Casper RC, Jabine LN. An eight-year follow-up: outcome from adolescent compared to adult onset anorexia nervosa. J Youth Adolesc. 1996; 25:499-517.

11. Calderon R, Vander Stoep A, Collett B, Garrison MM, Toth K. Inpatients with eating disorders: demographic, diagnostic and service characteristics from a Nationwide Pediatric Sample. Int J Eat Disord. 2007;40(7):622-8.

12. Wiseman CV, Sunday SR, Klapper F, Harris WA, Halmi KA. Changing patterns of hospitalization in eating disorder patients. Int J Eat Disord. 2001;30(1):69-74.

13. Chu ES, Gaudiani JL, Mascolo M, Statland B, Sabel A, Carroll K, et al. ACUTE center for eating disorders. J Hosp Med. 2012;7(4):340-4.

14. Gajewska M, Goryński P, Boguszewska L, Sowińska M. Hospitalization of people with eating disorders in Polish general and psychiatric hospitals in years 2003-2007. Przegl Epidemiol. 2009;63(4):571-7.

15. Alves CF0, Ribas VR, Alves EVR, Viana MT, Ribas RMG, Junior LPM, et al. Uma breve história da reforma psiquiátrica. Neurobiologia. 2009;72(1):96

16. Appolinario JC, Maya T. Serviços de transtornos alimentares no Brasil e no mundo. In: Nunes MA, Apolinário JC, Galvão AN, Coutinho W, editores. Transtornos alimentares e obesidade. 2a ed. Porto Alegre: Artmed; 2006.

17. Striegel-Moore RH, Leslie D, Petrill SA, Garvin V, Rosenheck RA. One year use and cost of inpatient and outpatient services among female and male patients with eating disorder: evidence from a National Database on Health Insurance Claims. Int J Eat Disord. 2000;27(4):381-9.

18. Salzano FT, Cordás TA. Hospital-dia (HD) para transtornos alimentares: revisão da literatura e primeiras impressões do HD do ambulatório de bulimia e transtornos alimentares do IPqHCFMUSP (AMBULIM). Rev Psiq Clín. 2003;30(3):86-94.

19. Zeeck A, Weber S, Sandholz A, Wetzler-Burmeister E, Wirsching M, Scheidt CE, et al. Inpatient versus day treatment for bulimia nervosa: results of a one-year follow-up. Psychother Psychosom. 2009;78(5):317-9.

20. Schwartz BI, Mansbach JM, Marion JG, Katzman DK, Forman SF. Variations in admission practices for adolescents with anorexia nervosa: a North American sample. J Adolesc Health. 2008:43(5):425-31.

21. Lopez-de-Andres A, Carrasco-Garrido P, Hernández-Barrera V, Gil-de-Miguel A, JiménezTrujillo I, Jiménez-García R. Hospitalization trends in Spanish children and adolescents with eating disorders. Appetite. 2010;55(1):147-51.

22. Willer MG, Thuras $P$, Crow SJ. Implications of the changing use of hospitalization. Am J Psychiatry. 2005;162(12):2374-6.

23. Gowers SG, Clark A, Roberts C, Griffiths A, Edwards V, Bryan C, et al. Clinical effectiveness of treatments for anorexia nervosa in adolescents. Br J Psychiatry. 2007;191:427-35.

24. Long CG, Kirsty-Anne F, Hollin CR. Treatment of chronic anorexia nervosa: a 4-year follow-up of adult patients treated in an acute inpatient setting. Clin Psychol Psychother. 2012;19(1):1-13

25. Hudson Jl, Hiripi E, Pope HG Jr, Kessler RC. The Prevalence and correlates of eating disorders in the National Comorbidity Survey Replication. Biol Psychiatry. 2007;61(3):348-58.

26. Favaro A, Caregaro L, Tenconi E, Bosello R, Santonastaso P. Time trends in age at onset of anorexia nervosa and bulimia nervosa. J Clin Psychiatry. 2009;70(12):1715-21.

27. Schimdt E, Mata GF. Anorexia nervosa: uma revisão. Fractal Rev Psicol. 2008;20(2):387-400.

28. Morgan CM, Vecchiatti IR, Negrão AB. Etiologia dos transtornos alimentares: aspectos biológicos, psicológicos socioculturais. Rev Bras Psiquiatr. 2002;24(Suppl 3):18-23.

29. Weaver L, Liebman R. Treatment of anorexia nervosa in children and adolescents. Curr Psychiatry Rep. 2012;14(2):96-100. 